\title{
Apprentissage du FLE en Algérie: de l'analyse des représentations à la motivation des apprenants dans le milieu universitaire
}

\author{
Errime Khadraoui \\ Université Mostefa Benboulaid, Batna, Algérie \\ Errime@yahoo.fr \\ Riad Messaour \\ Université Mohamed Boudiaf, M’Sila, Algérie \\ riad.casaie@yahoo.fr
}

\begin{abstract}
Résumé
La dynamique motivationnelle est-elle tributaire de l'évolution des représentations ? Quel rapport existe-t-il entre les représentations des étudiants vis-à-vis de la langue/culture française et l'apprentissage de celle-ci ? C'est à ces questions que la présente recherche tente de répondre. Pour cela, nous avons, dans un premier temps, réalisé à travers une enquête par questionnaire, une étude comparative entre les représentations qu'ont les étudiants de première année licence du département de français (Université de Batna 2) de la langue/culture française au début et en fin de l'année ; notre objectif étant d'examiner l'effet de l'évolution de leurs représentations sur leurs motivations. Afin d'appuyer les résultats obtenus de l'analyse des questionnaires, nous avons, dans un second temps, effectué des entretiens semi-directifs avec leurs enseignants. Au terme de cette étude, nous avons constaté une évolution de la motivation des étudiants qui s'explique par l'évolution de leurs représentations vis-à-vis de la langue/culture cible.
\end{abstract}

Mots-clés: apprentissage du FLE, représentations, motivation, milieu universitaire

\begin{abstract}
Is motivational dynamics dependent on the evolution of representations? What is the relationship between students' representations of French language/culture and its learning? These are the questions that this study seeks to address. To do so, we have, first, carried out through a questionnaire survey, a comparative study about the
\end{abstract}


KHADRAOUI, Errime; Messaour, Riad - Apprentissage du FLE en Algérie... Para lá da tarefa: implicar os estudantes na aprendizagem de línguas estrangeiras no ensino superior. Porto: FLUP, 2019, pp. 208-225 DOI: https://doi.org/10.21747/9789898969217/paraa11

representations that the first-year undergraduate students of the French department (University of Batna 2) of French language and culture, at the beginning and at the end of the academic year. Our objective is to measure the effect of the evolution of their representations on their motivations. To support the results obtained from the analysis of the questionnaires, we then have carried out semi-structured interviews with their teachers. At the end of this study, we noticed an evolution of student motivation that can be explained by the evolution of their representations towards the target language and culture.

Keywords: learning French as a foreign language, representations, motivation, university environment

\section{1 - Introduction}

Multiples sont les facteurs qui interviennent quand il s'agit d'apprendre une langue étrangère. Nous parlons ici des facteurs cognitifs et affectifs. S'agissant des facteurs cognitifs, on distingue l'aptitude et l'intelligence, dont le rôle dans l'apprentissage d'une langue étrangère est démontré par de nombreuses études. Quant aux facteurs affectifs, opposés à tort aux premiers, ils recouvrent les termes suivants : “ attitude ", «motivation» et «personnalité ». Ces facteurs sont aussi importants que les facteurs cognitifs car ils constituent selon Rodriguez, Plax et Kearney (1996) des sources de motivation intrinsèque. Comme l'apprentissage des langues étrangères ne dépend plus seulement des facteurs cognitifs, les facteurs affectifs sont aujourd'hui devenus l'objet de beaucoup de travaux de recherche dans le domaine de la didactique des langues étrangères (Arnold, 2006).

Cette didactique a vu l'apparition de diverses méthodes et approches dont l'approche communicative. Celle-ci a rénové certaines pratiques enseignantes, vu ses principes qui ont considéré l'apprenant comme l'acteur principal de son apprentissage (Beacco et al., 2005). Dans cette perspective, l'enseignant est invité prendre en compte les représentations de ses apprenants vis-à-vis de la langue étrangère enseignée. Ces apprenants ne sont plus considérés comme des récipients vides car ils entreprennent leurs apprentissages avec des représentations qui conditionnent leur assimilation. Ce sont ces représentations qui retiennent notre attention dans le présent travail qui a pour objectif principal d'examiner l'influence des représentations des apprenants sur leur motivation envers l'apprentissage du français langue étrangère en milieu universitaire. 
KHADRAOUI, Errime; Messaour, Riad - Apprentissage du FLE en Algérie... Para lá da tarefa: implicar os estudantes na aprendizagem de línguas estrangeiras no ensino superior. Porto: FLUP, 2019, pp. 208-225 DOI: https://doi.org/10.21747/9789898969217/paraa11

Ce phénomène de motivation occupe, aujourd'hui, une place importante dans l'enseignement/apprentissage des langues étrangères. Dans cette perspective, nous tenterons, à travers ce travail, de répondre aux interrogations suivantes : Quel impact pourrait avoir les représentations sur la motivation des apprenants vis-à-vis de l'apprentissage du FLE ? La dynamique motivationnelle est-elle tributaire de l'évolution des représentations ? Quel rapport existe-t-il entre les représentations des étudiants et l'apprentissage du français langue étrangère ?

Pour résumer, cette étude porte essentiellement sur l'influence des facteurs affectifs sur l'apprentissage du français langue étrangère en milieu universitaire. Pour ce faire, nous allons, dans la partie suivante, mettre l'accent sur les deux termes qui les représentent : “la motivation » et «les représentations ». En psychologie, ces deux termes sont considérés comme deux phénomènes très divers, mais ils sont intimement liés dans le domaine de la didactique des langues étrangères.

\section{2 - La motivation}

Pour beaucoup de chercheurs et scientifiques, donner une définition à la motivation n’a jamais été aisé. Généralement, la notion de « motivation » est utilisée pour expliquer la variabilité du comportement humain, ce qui veut dire qu'on l'emploie pour expliquer pourquoi, dans quelques situations, l'individu agit d'une façon différente que dans d'autres situations (Beck, 2003, p. 4, 5, cité par Kremenjaš, 2013, p. 6). En effet, parfois, posséder des compétences développées et du «talent» ne suffise pas pour réussir, notamment quand le but à atteindre est loin. Pour Topalov (2011, p. 9), l'individu doit dans ce cas avoir un niveau élevé de motivation. Dans le milieu scolaire Viau définie cette dernière comme « [...] un état dynamique qui a ses origines dans les perceptions qu'un élève a de lui-même et de son environnement et qui l'incite à choisir une activité à s'y engager et à persévérer dans son accomplissement afin d'atteindre un but. 》 (1994, p.7)

Tout le monde s'accorde à dire que la motivation est l'un des facteurs essentiels à l'apprentissage d'une langue étrangère. En ce sens, Marie J. Myers précise que « l'élément primordial sous-jacent aux apprentissages est la question de la motivation. Des remarques relatives aux émotions ont été faites dans tous les cas. II est donc d'importance cruciale de faire aimer la langue et de rendre son apprentissage désirable 》 (2004, p. 25). La motivation et l'apprentissage sont donc deux concepts étroitement liés l'un à l'autre dans le sens où «il n'y a pas d'apprentissage possible sans une 
KHADRAOUI, Errime; Messaour, Riad - Apprentissage du FLE en Algérie... Para lá da tarefa: implicar os estudantes na aprendizagem de línguas estrangeiras no ensino superior. Porto: FLUP, 2019, pp. 208-225 DOI: https://doi.org/10.21747/9789898969217/paraa11

mobilisation, une implication, un engagement plus au moins important » (Bourgeois, 2011, p. 230).

Plusieurs études portant sur les principaux aspects de la motivation reprennent le point de vue de Bogaards qui la présente comme « une tendance spécifique vers telle catégorie d'objets » (1988, p. 51). En effet, comme tendance celle-ci peut se manifester à travers différents degrés d'intensité. S'agissant maintenant des théories portant sur la motivation dans le contexte d'apprentissage des langues étrangères, nous pouvons citer celle de Gardner et Lambert. Ceux-ci se sont inspirés des conceptions des sociobéhavioristes, notamment celles de Mower qui stipule que l'acquisition de la parole par l'enfant se fait par l'imitation des parents. En effet, la présence de ces derniers est synonyme de satisfaction des besoins (Bogaards, 1988, p. 53). Ce processus d'imitation conduisant à l'acquisition de la langue est qualifié par Mowerd d'« identification ». Cet auteur précise à propos de ce processus qu'il doit être orienté vers la communauté linguistique.

Pour leur part, Gardner et Lambert estiment que ce processus d'identification est à l'origine de la motivation à long terme qui est à la base de l'apprentissage d'une langue étrangère. Il est à signaler que si la motivation à court terme est nécessaire à la réussite de la réalisation des tâches scolaires, la motivation à long terme est indispensable à l'installation et au développement d'une compétence effective dans la langue étrangère. Toutefois, la signification de cette notion d'identification diffère d'un auteur à un autre. Effectivement, Mower par exemple la conçoit comme la "satisfaction des besoins physiques ", alors que Gardner et Lambert la mobilisent pour renvoyer aux besoins sociaux. En effet, ces auteurs canadiens considèrent que l'apprentissage d'une langue n'est pas envisagé comme une fin en soi, mais plutôt comme un moyen servant à intégrer les sujets apprenants à une communauté déterminée. Ils emploient la notion d'identification pour désigner le processus d'acquisition de la langue maternelle, pour ce qui est de l'apprentissage des langues étrangères, ces chercheurs préfèrent utiliser la notion de «motif intégratif ». Celui-ci est défini par Bogaards comme étant « la volonté de devenir membre d'un groupe ethnolinguistique » (1988, p. 53).

Cette divergence de conceptions a permis de distinguer entre la "motivation intégrative » et la « motivation instrumentale ». La première est relative à la volonté de l'apprenant de connaitre la communauté de la langue/culture cible. L'intérêt manifesté à l'égard de cette communauté est motivé par sa volonté d'intégrer cette dernière. Au contraire, lorsqu'il s'agit d'une valeur instrumentale/utilitaire de la langue cible, on est en 
KHADRAOUI, Errime; Messaour, Riad - Apprentissage du FLE en Algérie...

Para lá da tarefa: implicar os estudantes na aprendizagem de línguas estrangeiras no ensino superior.

Porto: FLUP, 2019, pp. 208-225

DOI: https://doi.org/10.21747/9789898969217/paraa11

présence d'une motivation instrumentale. En d'autres termes, l'apprenant vise, à travers l'apprentissage de la langue étrangère, un objectif communicationnel ou professionnel.

Nous tenons à préciser que cette vision a fait l'objet de nombreuses critiques. L'une de ces critiques est le fait qu'elle se fonde sur l'approche béhavioriste de l'acquisition des langues (imitation et le conditionnement), l'autre pointe le rapprochement entre deux situations totalement différentes. En effet, cette critique souligne que la notion de l'identification n'est pas la même selon qu'il s'agit du désir du jeune enfant de faire partie de l'environnement de ses parents ou de la volonté d'un apprenant d'intégrer volontairement une communauté linguistique pour diverses raisons. En dépit de ces critiques, la théorie de Gardner et Lambert constitue un apport considérable dans la mesure où elle a montré que, d'une part, la motivation dépend aussi bien de variables individuelles que sociales et, d'autre part, elle constitue un levier très important dans le processus de l'apprentissage des langues étrangères.

Dans une classe de langue, certains facteurs influent sur la motivation. Jelaković les énumère dans la citation suivante : " l'intérêt du travail et sa nouveauté, le sentiment de progresser et d'atteindre les résultats, la conscience qu'on est en train d'apprendre une nouvelle culture et d'enrichir sa perception du monde » (2014, p. 12). La motivation peut donc être influencée par plusieurs facteurs, selon ce qu'ils soient externes (milieu familial, société, salle de classe) ou internes (les besoins personnels, les projets), nous distinguons :

\section{1 - La motivation intrinsèque (intégrative)}

Ce type de motivation est défini comme étant « les forces qui incitent à effectuer des activités volontairement, par intérêt pour elle-même et pour le plaisir et la satisfaction que l'on en retire » (Roussel, 2000, p. 29). La motivation intrinsèque correspond à la fois au plaisir, à l'intérêt et à la satisfaction que peut avoir un apprenant en réalisant une activité. Cette dernière est donc réalisée sans aucune pression ni contrôle externe dans le sens où l'apprenant n'espère aucun compliment voire aucune récompense. Ces récompenses sont plutôt internes, appelées « récompenses psychiques » (Kremenjaš, 2013, p. 11).

Selon Biçer, les apprenants motivés intrinsèquement « sont des élèves consciencieux qui travaillent pour eux-mêmes » (2008, p. 12). L'apprentissage chez ces apprenants est de meilleure qualité par rapport à ceux qui sont extrinsèquement motivés (Biçer, 2008, p. 8). 
KHADRAOUI, Errime; Messaour, Riad - Apprentissage du FLE en Algérie... Para lá da tarefa: implicar os estudantes na aprendizagem de línguas estrangeiras no ensino superior. Porto: FLUP, 2019, pp. 208-225 DOI: https://doi.org/10.21747/9789898969217/paraa11

\section{2 - La motivation extrinsèque (instrumentale)}

Contrairement à la motivation intrinsèque, ce type de motivation se situe à l'extérieur du sujet. Ce sont les renforcements, les feed-back et les récompenses qui alimentent la motivation. L'élève effectue ici une activité pour en retirer un avantage ou pour retirer un désagrément (Roussel, 2000, p. 29). L'apprenant est motivé extrinsèquement soit pour chercher une récompense soit pour éviter des problèmes avec son enseignant ou ses parents. Autrement dit, les bonnes notes obtenues par l'apprenant ne révèlent pas forcément l'intérêt et la motivation de l'apprenant vis-à-vis de la matière enseignée. De même, l'apprentissage des apprenants motivés extrinsèquement est superficiel et de qualité inférieure; quant aux connaissances, elles ont tendance à s'effacer de la mémoire avec le temps (Biçer, 2008, p. 8).

\section{3 - Les représentations}

Le deuxième facteur que nous allons présenter est celui des représentations. Le domaine de la didactique des langues étrangères et plus particulièrement les recherches qui s'intéressent à l'acquisition des langues, considèrent les représentations comme une notion d'une grande importance. La notion de représentation se définit comme étant une "forme de connaissance courante, dite «de sens commun' », caractérisée par les propriétés suivantes : 1 . Elle est socialement élaborée et partagée ; 2 . Elle a une visée pratique d'organisation, de maitrise de l'environnement (matériel, social, idéel) et l'orientation des conduites et communications ; 3 . Elle concourt à l'établissement d'une vision de la réalité commune à un ensemble social (groupe, classe, etc.) ou culturel donné » (Jodelet, 1991, p. 668).

Les représentations que se font les sujets d'une langue, de ses spécificités, de son statut par rapport aux autres langues, influencent considérablement leurs stratégies d'apprentissage de cette même langue. A ce sujet, les nouvelles conceptions psychologiques relatives aux attitudes ou aux motivations individuelles sont désormais rattachées aux images et aux conceptions sociales. Moore (2005) nous explique que la manière dont ces représentations se manifestent dans les interactions correspond à l'identique à celle qui a permis leur intégration et leur construction par le sujet ou le groupe.

Dans le domaine des représentations sociales, la théorie qui fait l'unanimité est celle de Moscovici. Jelaković s'appuyant sur la conception de Moore (2005) conçoit ces représentations comme étant « (...) des éléments de la conscience sociale extérieurs aux individus et s'imposant à eux » (2014, p. 17). En réalité, afin d'appréhender leur 
KHADRAOUI, Errime; Messaour, Riad - Apprentissage du FLE en Algérie... Para lá da tarefa: implicar os estudantes na aprendizagem de línguas estrangeiras no ensino superior. Porto: FLUP, 2019, pp. 208-225 DOI: https://doi.org/10.21747/9789898969217/paraa11

contexte, les personnes se réfèrent à un cadre de référence, de normes sociales afin de se positionner et de développer leurs relations. II s'agit ainsi d'un savoir socialement construit et partagé qui contribue à l'élaboration sociale de la réalité. Cette représentation sociale qui se présente comme norme permet de classer les personnes, les objets, et les événements. Par ailleurs, elle participe également à I 'élaboration « des prototypes permettant d'évaluer d'autres objets » (Jelaković, 2014, p. 17). La littérature scientifique fait état de trois éléments interdépendants caractérisant les représentations. En effet, les spécialistes mettent en avant le fait qu'elles sont le produit de/par la communication, elles reconfigurent le réel, et la maitrise du milieu par son organisation.

Lorsqu'on introduit les représentations dans le processus d'enseignement/apprentissage des langues étrangères, celles-ci renvoient à des images à la fois sur la langue cible, sur les pays parlant cette langue, ainsi que sur les locuteurs ayant cette langue comme langue maternelle. Les chercheurs relèvent une corrélation prépondérante entre l'image que se fait un apprenant du pays, et de ses locuteurs et les représentations qu'il élabore concernant son apprentissage de la langue de ce pays (Castellotti, 1995, p. 32). En ce sens, Jelaković (2014) illustre son propos en citant l'exemple de la langue allemande qui est relativement difficile pour les apprenants français et les suisses. Cette difficulté est selon le même auteur due principalement à une image négative de l'Allemagne.

En somme, les représentations se construisent et se développent au sein d'une communauté grâce à une multitude de canaux tels que les mass-médias, les textes littéraires, les dépliants publicitaires... Par ailleurs, les représentations sont dynamiques, en d'autres termes, elles " s'échangent au cours des communications quotidiennes, s'élaborent, se revitalisent et se cristallisent » (Jelaković, 2014, p. 18).

\section{4 - Représentations et motivation : quels rapports ?}

Nous avons, dans la partie ci-dessus, défini les deux termes motivation et représentation de façon séparée. Nous tenterons désormais de présenter le lien existant entre eux dans le domaine de la didactique des langues étrangères. En effet, plusieurs travaux de recherche ont prouvé que les représentations que se font les apprenants d'une langue étrangère peuvent être la cause de leurs échecs scolaires, de leurs réussites ou encore de leurs motivations lors de l'apprentissage de celle-ci.

Les représentations sociales de l'apprenant à l'égard de l'apprentissage d'une langue reflètent sa façon de penser ainsi que sa démarche intellectuelle. Elles peuvent être favorables ou défavorables. C'est alors en se basant sur ses propres images, que 
KHADRAOUI, Errime; Messaour, Riad - Apprentissage du FLE en Algérie... Para lá da tarefa: implicar os estudantes na aprendizagem de línguas estrangeiras no ensino superior. Porto: FLUP, 2019, pp. 208-225 DOI: https://doi.org/10.21747/9789898969217/paraa11

l'apprenant choisit ses démarches et mobilise le schéma à suivre pour agir ou réagir durant le processus pédagogique en adoptant un processus interprétatif qui lui est personnel.

En admettant que la notion de motivation est étroitement liée à celle de représentation, l'influence de cette dernière sur la motivation n'est, pour beaucoup de chercheurs, plus à démontrer. En fait, si l'apprenant se fait une bonne image sur une langue étrangère, il sera plus motivé pour l'apprendre. Si, au contraire, il a une mauvaise image sur la langue, cela peut malheureusement le démotiver d'une manière partielle ou complète à apprendre cette langue. Pour résumer, être motivé à l'apprentissage dépend des représentations que peut avoir l'apprenant (Barré-De Miniac, 2006).

\section{5 - Méthodologie}

Notre recherche consiste à effectuer une étude comparative entre les représentations qu'ont les étudiants de première année licence du département de français de l'université de Batna 2 en Algérie au début et en fin de l'année. Afin de pouvoir apporter les éléments de réponse nécessaires à notre problématique de recherche, nous avons opté pour les instruments de recherche suivants :

\section{1 - Enquête par questionnaire}

Nous avons choisi d'adresser à nos enquêtés le même questionnaire au début et à la fin de l'année, et ce dans le but de nous permettre d'examiner l'évolution de leurs représentations et l'influence de celles-ci sur leurs motivations. Le questionnaire que nous avons élaboré est composé de 16 questions dont 10 questions fermées et 6 questions ouvertes. Les deux questionnaires sont presque identiques ; Nous avons remplacé la question portant sur le choix du cursus par la question suivante : «Avezvous participé à la première enquête ? " Cette question avait pour objectif d'écarter les réponses des étudiants n'ayant pas participé à la première enquête.

Les premières informations demandées étaient à dimension identitaire. Nous avons voulu nous faire une idée précise du public avec lequel nous comptons travailler. Nous avons donc posé des questions sur leur sexe et leur âge.

La deuxième partie du questionnaire est composée des questions concernant les représentations des étudiants vis-à-vis de la langue française et son apprentissage en général. Voici quelques questions : 
KHADRAOUI, Errime; Messaour, Riad - Apprentissage du FLE en Algérie... Para lá da tarefa: implicar os estudantes na aprendizagem de línguas estrangeiras no ensino superior.

Porto: FLUP, 2019, pp. 208-225 DOI: https://doi.org/10.21747/9789898969217/paraa11

«Aimez-vous la langue française ? Justifiez; Que pensez-vous de la langue française ? La langue française est pour vous : 1. Très facile à apprendre ; 2. Facile à apprendre ; 3. Moyen ; 4. Difficile à apprendre ; 5. Très difficile à apprendre »

Pour ce qui est de la troisième partie, elle était réservée aux questions portant sur leurs motivations à l'égard de l'apprentissage du français :

«Expliquez les motivations du choix de ce cursus ? Êtes-vous motivé pour apprendre le français? Justifiez? Comptez-vous poursuivre ce cursus (L'apprentissage de cette langue) ? Pendant les séances de cours vous êtes plutôt : 1 . Très intéressé ; 2 . Intéressé ; 3. Pas intéressé ; 4. Ennuyé »

\section{2 - Enquête par entretien}

Des entretiens semi-directifs ont été menés auprès de leurs enseignants. Ceux-ci ont été interrogés afin qu'ils nous renseignent sur la motivation de leurs étudiants. Nous tenons à préciser que notre entretien est à usage complémentaire dans le sens où il a pour objectif de compléter les données déjà collectées à partir de l'analyse comparative des deux questionnaires. Autrement dit, nous nous sommes servis des résultats de l'entretien pour voir si la motivation des étudiant a réellement changé au cours de l'année universitaire.

Dans leurs réponses, les enseignants se sont appuyés sur l'observation de la participation de leurs étudiants en classe ainsi que sur l'évolution de leurs résultats (notes obtenues) entre le premier et le deuxième semestre de l'année universitaire.

Nous avons au préalable préparé un guide d'entretien où les questions étaient principalement centrées sur la motivation de leurs étudiants. L'entretien a été mené auprès de 9 enseignants de première année Licence. Les enregistrements ont fait l'objet d'une analyse qualitative.

«A votre avis, vos étudiants de première année sont-ils motivés ? Avez-vous observé un changement au niveau de la motivation de vos étudiants entre le début et la fin de l'année ? Si oui, comment expliquez-vous ce changement ? Comment s'est-elle manifestée ? » 


\section{6 - Population et échantillon d'étude}

Notre recherche a eu lieu au département de français de l'université de Batna 2, située à l'est de l'Algérie. Nous avons interrogé au total 197 étudiants de première année licence répartis sur 4 groupes. Parmi les enquêtés, nous avons observé une nette majorité de filles puisqu'il y a 179 filles et seulement 18 garçons. La majorité des étudiants ont entre 18 et 25 ans et que 11 seulement avaient plus de 25 ans.

\section{7 - Principaux Résultats de l'analyse du 1er questionnaire}

Ce questionnaire a été, comme nous l'avons mentionné plus haut, adressé aux étudiants de première année du département de français au début de l'année.

\section{1 - Analyse des représentations des étudiants vis-à-vis de la langue française et son apprentissage}

Par la question « aimez-vous la langue française ? ", nous avons voulu connaitre les représentations des apprenants à l'égard de la langue française. L'analyse des réponses obtenues nous a révélé que $71 \%$ des étudiants ont déclaré qu'ils aiment la langue française. Bien que ce pourcentage soit élevé, ils ont qualifié cette dernière, à travers les justifications avancées, de « difficile », « complexe » et « ses règles grammaticales comportent beaucoup d'exceptions ». Les éléments complexes et difficiles évoqués par les étudiants relèvent essentiellement de l'aspect purement linguistique de la langue, ce qui révèle la nature de l'enseignement subi dans les phases précédant la formation universitaire. Cette affirmation de la complexité linguistique montre également que les apprenants ne disposent pas de suffisamment de connaissances sur la langue/culture française, ce qui explique leurs commentaires négatifs.

Ces représentations sont également confirmées par le pourcentage des étudiants $16 \%$ et $38 \%$ qui la jugent respectivement « difficile à apprendre » et « très difficile à apprendre ». Ce même point de vue est soutenu par $79 \%$ d'étudiants qui la considèrent "plus difficile que les autres langues », tandis que seul $21 \%$ la juge « plus facile que les autres langues ». Notons que la difficulté dont il est question dans cette comparaison est faite essentiellement par rapport à la langue anglaise, deuxième langue étrangère apprise par les étudiants dans le système éducatif algérien. Cette langue est apprise en parallèle avec la langue française, ce qui justifie cette perspective comparative chez les étudiants.

De plus, ils font état d'une vision complexe et parfois paradoxale de la langue française. Grâce à la question «Que pensez-vous de la langue française ? », nous 
KHADRAOUI, Errime; Messaour, Riad - Apprentissage du FLE en Algérie... Para lá da tarefa: implicar os estudantes na aprendizagem de línguas estrangeiras no ensino superior. Porto: FLUP, 2019, pp. 208-225 DOI: https://doi.org/10.21747/9789898969217/paraa11

avons pu dégager les représentations liées aux traits linguistiques, culturels, historiques ainsi qu'esthétiques de la langue française. Cela s'est traduit d'une part par «les difficultés, et la complexité de son enseignement ", et du fait qu'elle est "démodée» et «dépassée par l'anglais dans le monde» d'autre part par "sa beauté », "son statut dans le monde», et «la culture qu'elle véhicule». Toutefois, le nombre de ceux ayant une conception positive de cette langue est minime (17\%).

Le premier enseignement que nous pouvons tirer de cette première série de questions est que nos enquêtés ont de la langue/culture française et de son enseignement/apprentissage une conception relativement négative. En effet, leurs réponses trahissent une forme d'appréhension vis-à-vis de cette langue.

\section{2 - Analyse de la motivation des étudiants à l'égard de l'apprentissage du français}

En voulant savoir si le choix du cursus est dû à un choix personnel, à l'orientation ${ }^{1}$ ou bien à un choix de la famille, Nous avons constaté que le choix d'étudier cette langue n'est pas toujours le résultat d'un choix personnel. En effet, $39 \%$ des étudiants soit 77 ont déclaré qu'ils auraient aimé choisir une autre langue ou un autre cursus. Ces chiffres nous permettent d'affirmer qu'un nombre conséquent d'étudiants a subi le choix du cursus universitaire. Ce qui pourrait avoir des répercussions négatives quant à leur implication dans la formation.

Pour les $61 \%$ restants, le choix de cette langue est pleinement assumé. Ils mettent en avant les raisons suivantes que nous avons regroupées après avoir effectué une analyse qualitative des réponses obtenues en appliquant la méthode de catégorisation des idées exprimées par les participants :

\footnotetext{
${ }^{1}$ En Algérie, l'orientation s'effectue en fonction de la moyenne du BAC ainsi que les notes de certaines matières.
} 


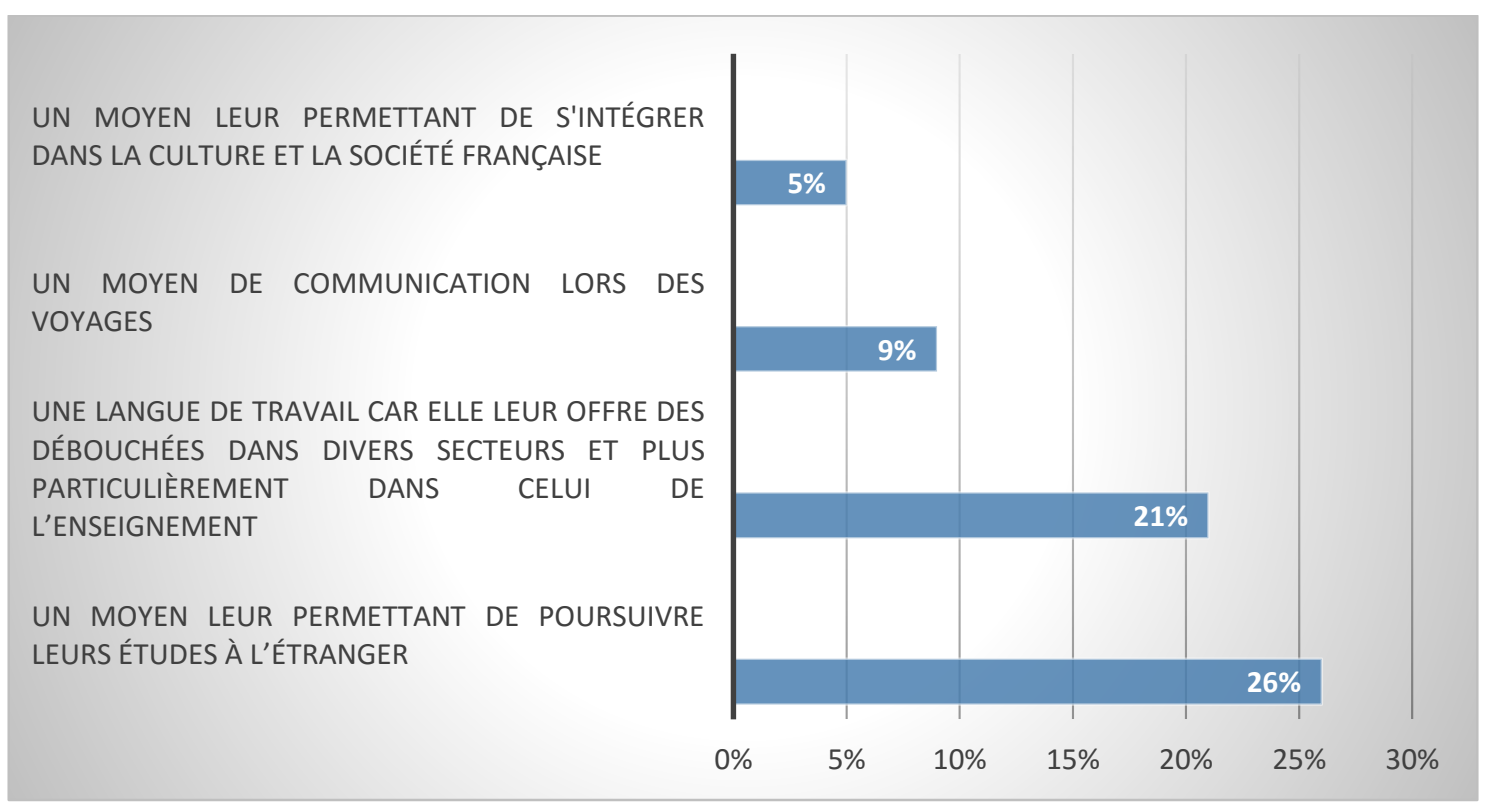

Figure 12. Motivations du choix de la langue française

Les pourcentages affichés dans le secteur graphique ci-dessus montrent que les principales raisons évoquées par ces étudiants relèvent d'une motivation instrumentale. En effet, pour $26 \%$ des étudiants le français est un moyen qui leur permet de poursuivre leurs études à l'étranger, de décrocher un travail dans divers secteurs et plus particulièrement dans celui de l'enseignement et de faciliter la communication lors des voyages. Les extraits suivants, tirés de notre corpus, illustrent ce type de motivation :

E 5 : J'ai choisi cette langue (français) pour devenir enseignant E 12: Je compte voyager dans l'avenir pour peut-être m'installer en France ou au Québec pour faire un master

Pour ce qui est de la motivation intégrative, elle est nettement inférieure par rapport la motivation instrumentale. Elle est exprimée par nos étudiants par à la fois leur souhait de s'intégrer dans la culture et la société française et leur propre plaisir d'apprendre la langue française. Dans ce qui suit les réponses de nos enquêtés à propos des motivations qui les ont menées à choisir l'enseignement de cette langue :

E 14: J'ai choisi cette langue parce qu'elle est belle, comparée aux autres langues, je la trouve sublime. Elle est classe.

E 42: Ce cursus va me permettre de mieux comprendre l'Histoire et la culture de la France et des français. 
KHADRAOUI, Errime; Messaour, Riad - Apprentissage du FLE en Algérie... Para lá da tarefa: implicar os estudantes na aprendizagem de línguas estrangeiras no ensino superior. Porto: FLUP, 2019, pp. 208-225 DOI: https://doi.org/10.21747/9789898969217/paraa11

S'agissant de la motivation des étudiants à s'impliquer dans ce cursus (langue), 43\% des étudiants indiquent ne pas être motivés pour apprendre cette langue. Ces étudiants justifient leur manque de motivation par les difficultés qu'ils rencontrent dans l'apprentissage de celle-ci. II apparait donc que le sentiment d'incompétence de ces étudiants est la principale source de l'amotivation (Ryan \& Deci, 2000):

E 30 : J'ai du mal à comprendre presque tous les concepts vus dans les matières.

E 9: C'est une langue très difficile. Elle a beaucoup d'exceptions.

Le nombre d'étudiants (78) soit $39.59 \%$ qui avouent ne pas s'intéresser aux enseignements de langue est très important. Ce désintérêt se manifeste dans les taux de participation relevés qui sont faibles. Quant à la participation, 89\% des étudiants déclarant ne pas participer lors des cours, et justifient cela par « la peur de se tromper devant les autres », "la timidité », et « la complexité des notions abordées en cours ».

Enfin, plus d'un tiers des répondants ne semble pas sûr de vouloir poursuivre l'apprentissage de la langue française. La justification fournie par ces derniers met en avant le fait que la langue française soit une « langue très difficile » et qu' " elle comporte plein d'exception ce qui complique son apprentissage ». De plus, Certains d'entre eux évoquent le fait qu' « ils n'ont pas choisi ce cursus ».

Pour résumer, la lecture de l'ensemble des résultats de ce premier questionnaire révèle qu'un nombre non négligeable d'étudiants de première année ont de la langue française ainsi que de son enseignement/apprentissage des représentations plutôt négatives. Ces dernières semblent constituer un frein à la motivation et à l'implication des étudiants.

\section{8 - Principaux résultats de l'analyse du $2 \mathrm{e}$ questionnaire}

Nous avons administré le même questionnaire aux mêmes étudiants, mais cette foisci en fin d'année. Nous tenons à préciser que cette partie consiste à présenter les résultats de l'analyse de ce questionnaire tout en les confrontant à ceux du premier questionnaire. 


\section{1 - Analyse des représentations des étudiants vis-à-vis de la langue française et de son apprentissage}

Avant toute chose, nous tenons à préciser que l'analyse de l'évolution des représentations des étudiants repose à la fois sur l'augmentation ou la diminution de certains chiffres auxquels nous sommes parvenus lors de la première enquête, mais aussi sur le changement de certaines conceptions jugées négatives dans l'analyse des représentations du premier questionnaire. Ces conceptions sont perceptibles à travers les commentaires formulés par les apprenants en répondant aux questions posées.

$80 \%$ des étudiants affirment aimer la langue française. Comparés aux chiffres indiqués au début de l'année, ceux-ci montrent que certains étudiants semblent reconsidérer leurs sentiments vis-à-vis de la langue française. Bien que le pourcentage $(69 \%)$ indiquant que l'enseignement de la langue française est «plus difficile que les autres » soit inférieur à celui noté en début d'année (79\%), nos étudiants continuent à la percevoir comme étant une langue «plus difficile que les autres ».

En ce qui concerne leurs représentations envers la langue/culture française d'une manière générale, nos enquêtés estiment que la langue française est une langue qui a un certain " charme » et une certaine « classe " car, selon leurs réponses, " elle fut enseignée à la noblesse européenne dans tous les royaumes d'Europe ». De plus, elle est synonyme « d'émancipation », "langue de communication internationale », et « une langue d'ouverture sur les autres ». A cet égard, le taux des réponses à connotation positive est passé de $17 \%$ à $52 \%$. Nous avons également relevé l'absence de toute domination de la langue anglaise dans les comparaisons. Au contraire, dix-neuf étudiants ont vanté les mérites de « la rigidité des règles grammaticales de la langue française » qui, selon eux, permet de la préserver de toute «transgression » qui lui conférerait une certaine « souplesse » reprochée, par ailleurs, à la langue anglaise.

Par ailleurs, un bon nombre d'étudiants (35\%) ont associé à la langue française des stéréotypes valorisants qui renvoient tantôt à sa civilisation séculaire « elle existe depuis des siècles » et tantôt à l'art et à la culture qu'elle véhicule " littérature riche », "le développement du classicisme ». La simple présence de ces éléments dans les réponses des étudiants peut être considérée comme étant une prise de conscience de quelques faits se rapportant à la langue/culture française et par conséquent une évolution des représentations de certains étudiants surtout qu'ils ne les ont pas évoqués en début d'année.

Tout compte fait, la lecture des différents résultats du deuxième questionnaire nous a permis de noter une certaine évolution des représentations des étudiants vis-à-vis de 
KHADRAOUI, Errime; Messaour, Riad - Apprentissage du FLE en Algérie... Para lá da tarefa: implicar os estudantes na aprendizagem de línguas estrangeiras no ensino superior. Porto: FLUP, 2019, pp. 208-225 DOI: https://doi.org/10.21747/9789898969217/paraa11

la langue/culture française. Effectivement, cette évolution s'est traduite chez ces étudiants par un changement d'une conception relativement négative à une conception qui pourrait être qualifiée de positive, tant les réponses des étudiants.

\section{2 - Analyse de la motivation des étudiants à l'égard de l'apprentissage du français}

$65 \%$ des répondants affirment être motivés à apprendre et à maitriser cette langue. II est à signaler que les explications avancées par nos enquêtés insistent à la fois sur l'intensité et la fréquence de leurs motivations. En effet, ils ont indiqué qu'ils sont « plus » et « souvent » motivés. Ces pourcentages indiquent que le désintéressement durant les séances de cours a reculé, en effet, il est passé de $40 \%$ au début de l'année à $28 \%$ en fin de l'année.

Nous avons également constaté que le taux de participation est passé de $11 \%$ au début de l'année universitaire à $22 \%$ en fin d'année. La question suivante " Comptezvous poursuivre ce cursus (l'apprentissage de cette langue) ? " nous a permis de remarquer que les enseignements dispensés tout au long de l'année ont permis à un bon nombre d'étudiants qui étaient jusque-là indécis de se prononcer par rapport à leur avenir. En effet, 10\% des indécis se sont prononcés pour la poursuite de ce même cursus alors que $02 \%$ comptent quant à eux s'orienter vers d'autres horizons.

\section{9 - Principaux résultats de l'analyse de l'entretien}

Tous les enseignants s'accordent à dire que d'une part, les étudiants ne sont pas tout le temps motivés et d'autre part, ils ne sont pas tous motivés de la même manière. Les enseignants interrogés sont unanimes sur le fait que la motivation manifestée par un grand nombre d'étudiants de première année vient du fait qu'ils sont « excités » et « grisés » par leur passage du lycée à l'université. En effet, ils expliquent cette motivation par l'envie de découvrir le milieu universitaire en général et l'organisation des matières en particulier.

Ils indiquent qu'au début de l'année et plus précisément durant les premières séances (les trois premières séances), la quasi-totalité des étudiants assistent assidument aux cours et manifestent une attention particulière pour les enseignements dispensés. Cependant, cet état ne dure pas longtemps et petit à petit le taux d'assiduité diminue. Les enseignants universitaires notent également une forme de désintéressement chez un grand nombre d'étudiants. Selon ces mêmes enseignants, cette situation les a contraints à questionner leurs étudiants à propos de ce manque d'implication 
KHADRAOUI, Errime; Messaour, Riad - Apprentissage du FLE en Algérie... Para lá da tarefa: implicar os estudantes na aprendizagem de línguas estrangeiras no ensino superior. Porto: FLUP, 2019, pp. 208-225 DOI: https://doi.org/10.21747/9789898969217/paraa11

(motivation). A ce sujet, les principaux intéressés évoquent « les difficultés inhérentes à cette langue », «leur volonté de changer le cursus en fin d'année car ce n'était pas leur premier choix ».

Ces enseignants expliquent l'a-motivation de leurs étudiants par les représentations parfois erronées qu'ont ces derniers de la langue cible. Pour remédier à ce problème, ils affirment qu'ils entreprennent tout un travail sur les représentations des étudiants. Toutefois, ils précisent que leurs démarches sont intégrées aux cours. Ainsi, ils attribuent le regain d'intérêt et la motivation exprimée à l'égard de la langue française, à l'évolution des représentations de certains étudiants.

La motivation se manifeste, d'après les enseignants universitaires, par une « hausse notable de l'assiduité et de la participation », " recrudescence du degré d'implication et de la prise d'initiative dans les travaux », ainsi que «l'amélioration des notes ».

\section{0 - Conclusion}

Au terme de cette étude, nous avons constaté une évolution de la motivation de certains étudiants qui s'explique par l'évolution de leurs représentations vis-à-vis de la langue/culture françaises. II apparait donc que la motivation de l'apprentissage d'une langue étrangère est en partie tributaire des représentations que font les apprenants de cette langue.

II est à signaler que les étudiants concernés par notre étude n'ont pas justifié leurs manques d'implication par des raisons historiques et cela malgré qu'un certain nombre de chercheurs algériens estiment que les représentations des faits historiques (la colonisation) sont un frein à la motivation et un facteur entravant considérablement l'apprentissage de la langue française. En effet, Benamar explique l'impact de ces représentations en affirmant que « (...) Le rejet du français dû à une rancune historique héritée de père en fils augmente la démotivation de l'apprenant, et l'éloignement de la matrice linguistique constituant un obstacle de taille (...) » (2009, p. 279).

\section{Références bibliographiques}

Arnold, J. (2006). Comment les facteurs affectifs influencent-ils l'apprentissage d'une langue étrangère ?. Études de linguistique appliquée, 144(4), 407-425. Récupéré de https://www.cairn.info/revue-ela-2006-4-page-407.htm.

Barré-De Miniac, C. (2000). Le rapport à l'écriture, Aspects théoriques et didactiques. Villeneuve d'Ascq (Nord): Presses universitaires du Septentrion. 
KHADRAOUI, Errime; Messaour, Riad - Apprentissage du FLE en Algérie... Para lá da tarefa: implicar os estudantes na aprendizagem de línguas estrangeiras no ensino superior. Porto: FLUP, 2019, pp. 208-225 DOI: https://doi.org/10.21747/9789898969217/paraa11

Beacco, J.-C., Chiss, J.-L., Cicurel, F. \& Véronique, D. (dir.). (2005). Les cultures éducatives et linguistiques dans l'enseignement des langues. Paris: Presses universitaires de France.

Benamar, N. (2009). L'enseignement/Apprentissage du FLE: Obstacles et Perspectives. Synergies Algérie, 7, 277-288.

Biçer, O. (2008). La motivation des élèves dans l'apprentissage des langues étrangères, les influences de la motivation sur la réussite dans l'apprentissage du français langue étrangère- (thèse de maitrise). Université de Gazi, Ankara. Récupéré de http://www.belgeler.com/blg/1b61/la-motivation-des-Ives-dans-l-apprentissage-deslangues-trangres-les-influences-de-la-motivation-sur-la-reussite-dans-l-apprentissagedu-franais-langue-trangre-yabanci-dil-eitiminde-renci-motivasyonu-fransizca-eitiminderenci-motivasyonunun-baariya-etkileri.

Bogaards, P. (1988). Aptitude et affectivité dans l'apprentissage des langues étrangères. Paris: Hatier-Crédif.

Bourgeois, É. (2011). La motivation à apprendre. In É. Bourgeois (Eds.), Apprendre et faire apprendre (233-253). Paris: Presses Universitaires de France.

Castelloti, V. (1995). La formation des enseignants de langue. Paris: CLE International.

Jelaković, M. (2014). Motivation et attitudes des apprenants en langue française (Mémoire de master). Université de Zegreb, Zegreb. Récupéré de http://darhiv.ffzg.unizg.hr/id/eprint/5141/1/Mateja\%20Jelakovi\%C4\%87\%20\%20Diplomski\%20rad.pdf

Jodelet, D. (1991). Représentation sociale. Grand dictionnaire de la psychologie. Paris: Larousse. 668-672.

Kremenjaš, K. (2013). Motivation pour l'apprentissage du français langue étrangère (Mémoire de master). Université de Zagreb: Zagreb.

Moore, D. (coord.) (2005). Les représentations des langues et leur apprentissage : Références, modèles, données et méthodes. Paris: Didier.

Myers, J. (2004). Modalités d'apprentissage d'une langue seconde. Bruxelles: De Boeck Université. 
KHADRAOUI, Errime; Messaour, Riad - Apprentissage du FLE en Algérie... Para lá da tarefa: implicar os estudantes na aprendizagem de línguas estrangeiras no ensino superior. Porto: FLUP, 2019, pp. 208-225 DOI: https://doi.org/10.21747/9789898969217/paraa11

Rodriguez, J., Plax, T. \& Kearney, P. (1996). Clarifying the relationship between teacher nonverbal immediacy and student cognitive learning: affective learning as the central causal mediator. communication education, 45, 294-305.

Roussel, P. (2000). La motivation au travail - concept et théories. Les notes du LIRHE, Université Toulouse I - Sciences Sociales.

Ryan, R.M., Deci, E.L. (2000). Intrinsic and Extrinsic Motivation : Classic Definitions and New Directions. Contemporary Educational Psychology, 25, 54-67. Récupéré de http://www.sciencedirect.com/science/article/pii/S0361476X99910202

Viau, R. (1994). La motivation en contexte scolaire. Québec: Les Éditions du Renouveau Pédagogique Inc. 